\title{
Events at the surface of the cell
}

\section{Cell division requires a simple external signal to be converted into a complex series of internal events. Attention to that process would help more than further oncogene sequences in understanding cancer.}

AMonc; speakers at last month's EMBO Symposium on Growth Factors, Receptors and Oncogenes (Heidelberg, 16-19 September) the most common currency was a figure along the lines of that shown here. The exact version differed according to the interests and beliefs of individual speakers. But all attested to the growing recognition that the hard facts of molecular biology have, sooner rather than later, to be integrated with cell physiology and even plain old biochemistry if they are not to be biologically sterile.

This is nowhere more so than in the case of oncogencs, where molecular information on gene and protein sequences has far outstripped understanding of how the proteins are involved in tumorigenesis. And exactly the same can be said of the genes from which oncogenes are derived (by mutation) and whose proteins are involved in normally regulated cell growth. Thus we know the sequences of many normal growth factors, a few of their cellsurface receptors and a number of oncogenes that are known or suspected to be mutants of growth factor or receptor genes, but much less about how the signal generated by a growth factor meeting its receptor on the exterior surface of a cell's membrane is converted into internal instructions that lead to cell division.

At least something is known, of the initial events. Thus the binding of some growth factors, including platelet-derived and epidermal growth factors (PDGF and EGF), to their receptors, activates a tyrosine kinase (TK) activity on the receptors' internal tail. Some ligand-receptor complexes, including EGF-receptor, are internalized. The PDGF-receptor complex, unlike the EGF-receptor complex, activates phospholipase C (PLC), which generates inositol trisphosphate $\left(\mathrm{IP}_{3}\right)$ and diacylglycerol (DG). IP, leads to the release of intracellularly stored calcium ions $\left(\mathrm{Ca}^{2+}\right)$; DG activates protein kinase $\mathrm{C}$ (PKC), which seems to trigger $\mathrm{Na}^{+} / \mathrm{H}^{+}$exchange, accounting for the transient increase in $p \mathrm{H}$ that characterizes growth factor-stimulated cells. But some of these links are poorly understood and there are many others to be forged if the initially observed events in a stimulated cell are to be explained, let alone the end result of regulated or, in cancer, unregulated cell division.

One particular puzzle is where the protein products of the ras genes fit in. These proteins are located in the cell surface. bind GTP and hydrolyse it. Certain mutations convert the genes into oncogenes, the protein products of which are much less good at hydrolysing GTP. With the collapse (see Nature News and Views 5 September, p.16) of the idea that ras proteins might be the GTP binding proteins (G) that convert a hormonal signal at the cell surface into the production of cyclic AMP by adenylate cyclase (AC), attention at Heidelberg was turned to the possibility that ras proteins (RAS) are the link between receptors, such as that for PDGF, and phospholipase C (PLC). But so far it is hopes rather than evidence that keep that idea afloat.

Whereas the rise in internal $\mathrm{Ca}^{2+}$ that rapidly follows PDGF stimulation of a cell seems to be a consequence of the release of internally stored $\mathrm{Ca}^{2+}$, that following EGF stimulation is the result of an inward flux of extracellular $\mathrm{Ca}^{2+}$, according to $\mathrm{W}$. H. Moolenaar of the Hubrecht Laboratory in Utrecht. By generating monoclonal antibodies to the EGF receptor that

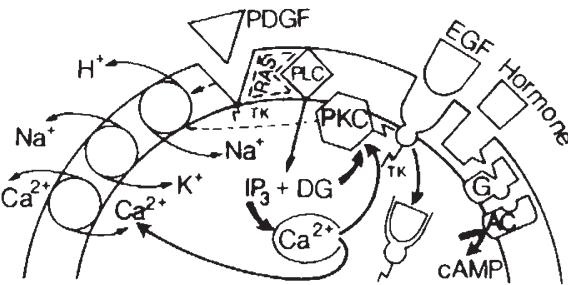

Initial events at the surface of a stimulated cell.

mimic EGF in terms of stimulating TK but not in raising internal $\mathrm{Ca}^{2+}$, he has neatly dissociated the two responses. The EGF receptor was the focus of attention by speakers from several laboratories, in part because it provides a good example of receptor 'transmodulation'. In this example, the affinity of EGF receptor for EGF is decreased by the binding of PDGF to its own receptor. It seems that the mechanism hinges on PKC which, activated by PDGF binding, phosphorylates EGF receptor on a serine residue that lies just on the cytoplasmic side of the cell's membrane. (How that decreases the affinity of the extracellular binding domain of the receptor or, for that matter, how EGF binding activates the intracellular TK, are pressing questions.) A contrasting example of transmodulation is the binding of insulin to adipocytes. This increases the number of insulin-like growth factor receptors on the cell surface by decreasing their phosphorylation, which inhibits their internalization, suggested M.P. Czech of the University of Massachusetts.

Whereas there is at least some understanding of the initial events mediated at or via the surface of a stimulated cell, next to nothing is known about the process by which later events are induced. For now. the emphasis is on finding out which genes, and in what order, are induced by stimulation. In that respect, a fascinating new turn of events, described by C.D. Stiles of the Dana-Faber Cancer Institute in Boston, is the increase in $\beta$-interferon and $2^{\prime}, 5^{\prime}$ - oligoadenylate synthetase gene transcripts after the stimulation of fibroblasts by PDGF, although considerably later in time than the previously documented induction of $m y c$ and fos (which are both implicated as oncogenes in other circumstances). Interferon and the synthetase are well known to be induced by viruses and are thought to mediate the antiviral response of cells, with the synthetase generating $2^{\prime}, 5^{\prime}$-adenylate which then activates a ribonuclease that is supposed to have a specificity for viral messenger RNA. Stiles suggests that the specificity is broader and perhaps includes $m y c$, fos and other messenger RNAs that must only be allowed a transient appearance in stimulated cells if their growth is to be normally regulated. In complementary experiments reported by Stiles, vesicular stomatitis virus or poly $(\mathrm{I}: \mathrm{C})$ induced $m y c$ and fos before interferon and the synthetase, and in much the same manner as PDGF.

In fully (serum) stimulated cells there are perhaps 100 induced.genes according to D. Nathans of Johns Hopkins University. Either luck or brute force will be needed to identify the key members of such a large set. Key member or not, one of 100 genes is of considerable interest in that it encodes a new hormone, proliferin, which is related by sequence to prolactin. In mouse tissues, a proliferin-like protein is found only in the placenta, which contains messenger RNAs for two proliferinlike peptides.

With the emergence of new hormones and reports of new receptors (several laboratories have characterized a receptor that is closely related to the EGF receptor, but none has found its ligand), oncogenes (including one that contains a large segment of a tropomyosin gene) and inositol phosphates (notably a 1,3,4-isomer of the usual $\left.1,4,5-\mathrm{IP}_{3}\right)$, the likelihood is that any figure of the type shown is destined soon to become a palimpsest. Peter Newmark 